\title{
RET kinase alterations in targeted cancer therapy
}

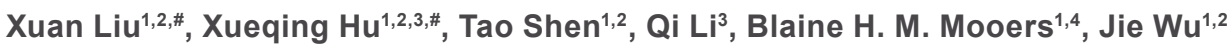 \\ 'Peggy and Charles Stephenson Cancer Center, University of Oklahoma Health Sciences Center, Oklahoma City, OK 73104, \\ USA. \\ 2Department of Pathology, University of Oklahoma Health Sciences Center, Oklahoma City, OK 73104, USA. \\ ${ }^{3}$ Department of Medical Oncology and Cancer Institute, ShuGuang Hospital, Shanghai University of Traditional Chinese \\ Medicine, Shanghai 201203, China. \\ ${ }^{4}$ Department of Biochemistry and Molecular Biology, University of Oklahoma Health Sciences Center, Oklahoma City, OK \\ 73104, USA. \\ \#Authors contributed equally.
}

Correspondence to: Prof. Jie Wu, Peggy and Charles Stephenson Cancer Center, University of Oklahoma Health Sciences Center, 975 NE 10th Street, BRC413, Oklahoma City, OK 73104, USA.E-mail: jie-wu@ouhsc.edu

How to cite this article: Liu X, Hu X, Shen T, Li Q, Mooers BHM, Wu J. RET kinase alterations in targeted cancer therapy. Cancer Drug Resist2020;3:472-81. http://dx.doi.org/10.20517/cdr.2020.15

Received: 29 Feb 2020 First Decision: 26 Mar 2020 Revised: 26 Mar 2020 Accepted: 31 Mar 2020 Available online: 11 May 2020

Science Editor: Lee M. Graves Copy Editor: Jing-Wen Zhang Production Editor: Tian Zhang

\begin{abstract}
The rearranged during transfection (RET) gene encodes a protein tyrosine kinase. RET alterations by point mutations and gene fusions were found in diverse cancers. RET fusions allow abnormal expression and activation of the oncogenic kinase, whereas only a few of RET point mutations found in human cancers are known oncogenic drivers. Earlier studies of RET-targeted therapy utilized multi-targeted protein tyrosine kinase inhibitors (TKIs) with RET inhibitor activity. These multi-targeted TKIs often led to high-grade adverse events and were subject to resistance caused by the gatekeeper mutations. Recently, two potent and selective RET TKIs, pralsetinib (BLU667) and selpercatinib (LOXO-292), were developed. High response rates to these selective RET inhibitors across multiple forms of RET alterations in different types of cancers were observed in clinical trials, demonstrating the RET dependence in human cancers harboring these RET lesions. Pralsetinib and selpercatinib were effective in inhibiting RET ${ }^{\mathrm{V} 804 \mathrm{~L} / \mathrm{M}}$ gatekeeper mutants. However, adaptive mutations that cause resistance to pralsetinib or selpercatinib at the solvent front $\mathrm{RET}^{\mathrm{G} 810}$ residue have been found, pointing to the need for the development of the next-generation of RET TKIs.
\end{abstract}

Keywords: Rearranged during transfection, protein tyrosine kinase inhibitor, mutation, gene fusion, targeted therapy, acquired resistance

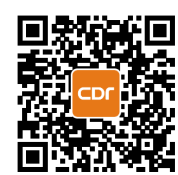




\section{INTRODUCTION}

Rearranged during transfection (RET) is a transmembrane receptor protein tyrosine kinase (PTK) activated normally by forming ternary complexes with its cognate ligands and co-receptors ${ }^{[1,2]}$. The four RET co-receptors, glial cell-derived neurotrophic factor (GDNF) receptor- $\alpha$ family proteins (GFR $\alpha 1-4)$, dictate ligand specificity. GDNF, neurturin, artemin, persephin, differentiation factor 15 (GDF15), and GDNF receptor- $\alpha$ (GFR $\alpha)$-like protein (GFRAL) are the currently known RET ligands ${ }^{[1-3]}$. Mutations and gene fusions could lead to aberrant dimerization, expression, and activation of the RET protein tyrosine kinase $^{[1,4,5]}$. RET point mutations were associated with medullary thyroid cancer $(\mathrm{MTC})^{[6]}$, whereas RET fusions were found in $20 \%$ of papillary thyroid carcinoma $(\mathrm{PTC})^{[7]}$ and $1 \%-2 \%$ of lung adenocarcinoma ${ }^{[8-11]}$. However, studies in the last few years have unraveled RET alterations in diverse cancer types ${ }^{[12-14]}$. While RET fusions are oncogenic, the functional significance of many RET point mutations remains to be characterized. RET alterations that result in activated RET kinase are targetable oncogenic events. Earlier efforts utilized multi-targeted TKIs with RET inhibitor activity ${ }^{[4,15-17]}$. These inhibitors are subject to resistance from mutations at the gatekeeper residue of the RET kinase domain. Recently, two highly potent and selective RET TKIs, pralsetinib ${ }^{[18]}$ and selpercatinib ${ }^{[19]}$, were developed. Preliminary results from clinical trials showed remarkable responses to these selective RET TKIs ${ }^{[20,21]}$. The high response rates of pralsetinib and selpercatinib unequivocally established RET as the therapeutic target in human cancers that harbor oncogenic RET-alterations. However, although the selective RET inhibitors are gatekeeper mutant-effective, acquired mutations at the solvent front residue have been found ${ }^{[22]}$. In this article, we critically review recent findings of RET alterations in human cancers, including both oncogenic and TKIadapted alterations, targeted therapy of RET-altered cancers, and raise issues that require further studies.

\section{RET ALTERATION IN CANCERS}

The RET proto-oncogene resides at human chromosome 10q11.21 and has 20 exons. The first 11 exons encode the extracellular and the transmembrane (exon 11) domains. Alternative splicing results in three proteins, RET51, RET9 and RET43, that differ in the lengths of carboxyl-terminal tails ${ }^{[23]}$. RET51 has 1114 amino acid residues [Figure 1A]. The longer RET51 has a stronger transformation activity than that of the shorter RET9 ${ }^{[11]}$. The RET extracellular domain has four atypical cadherin-like domains (CLD1-4) and a cysteine-rich domain (CRD) [Figure 1B]. The CRD has seven disulfide bonds. C630 and C634, located in the linker region between the CRD and the transmembrane domain, form another disulfide bone ${ }^{[3]}$. The extracellular domain forms a C-shaped clamp to bind a co-receptor and a ligand ${ }^{[3]}$. CLD1, CLD2, and CLD3 form the interacting face with the co-receptor, while CRD contacts both the co-receptor and the ligand. The ligand-induced dimerization of the receptor/co-receptor/ligand complex activates the receptor $^{[3,24]}$.

Oncogenic gain-of-function RET mutations were originally found in multiple endocrine neoplasia type 2 (MEN2). MEN2 has three clinical subtypes: MEN2A, MEN2B and familial medullary thyroid carcinoma (FMTC $)^{[1,23]}$. MEN2A-associated RET mutations occur most often in the extracellular regions, particularly disulfide bond-forming C609, C611, C618, C620, C630, and C634 residues located in the CRD domain and the region connecting the $\mathrm{CRD}$ and the transmembrane domains ${ }^{[1,4,23]}$. It is believed that mutations in these Cys residues disrupt the intramolecular disulfide bonds, allowing formation of abnormal intermolecular disulfide bonds, which lead to receptor dimerization and kinase activation. In the RET/co-receptor/ligand complexes (for instance, the RET/GFR $\alpha 1 / G D N F$ complex shown in Figure 1) ${ }^{[3]}$, the distance between two receptor molecules in any dimer of these complexes is too far for intermolecular disulfide bond formation between the Cys residues located within the CRD. Thus, based on the RET/co-receptor/ligand complex structures (PDB: 6Q2N, 6Q2S, 6Q2J, 6Q2R, 6Q2O), RET ligands appear to act as inhibitors for abnormal formation of intermolecular disulfide bonds between the C609, C611, C618 or C620 residues within the CRD. Thus, the ligand-independent dimerization via formation of intermolecular disulfide bond between 
A

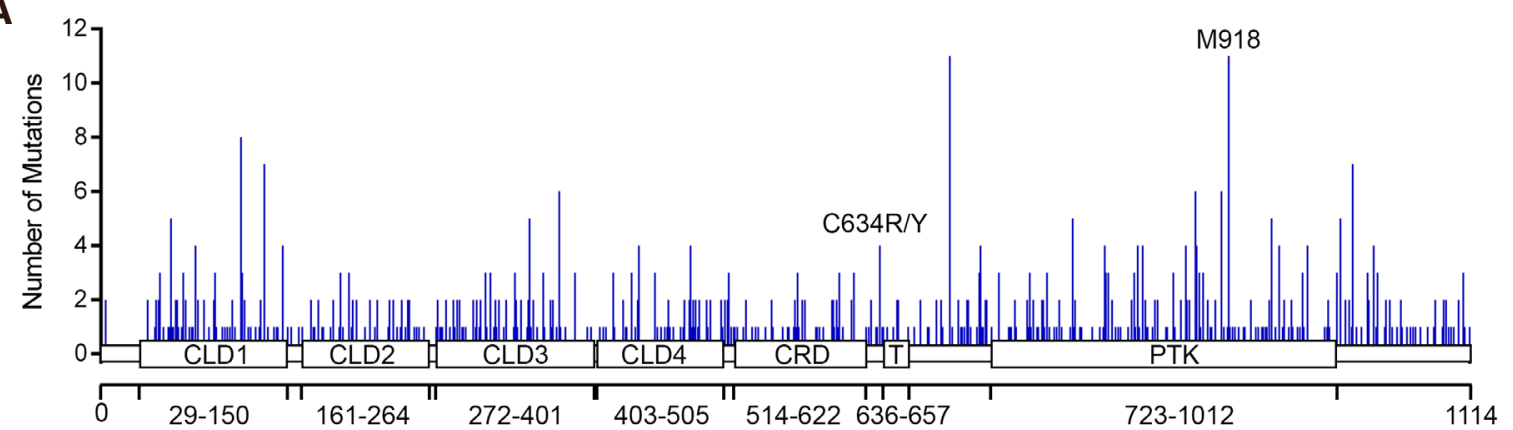

B

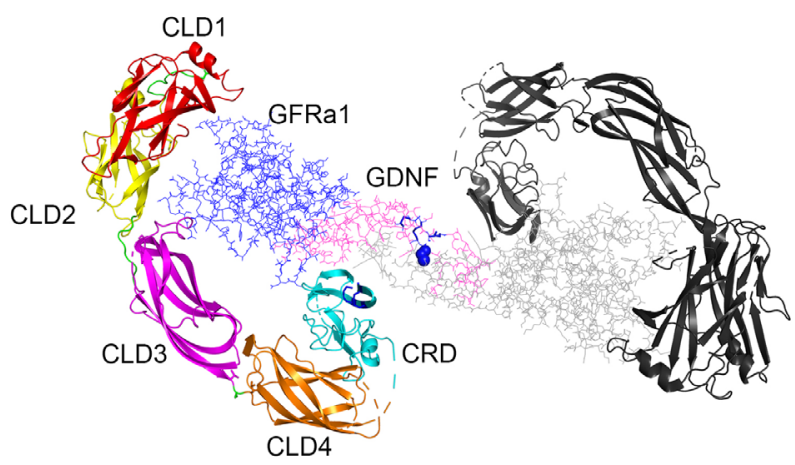

C

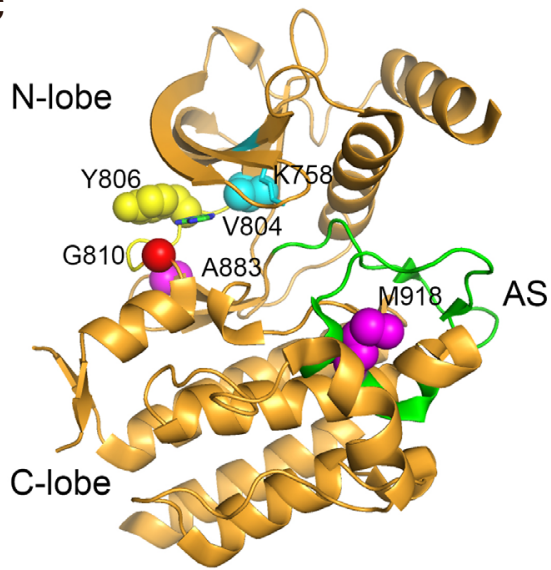

Figure 1. RET missense mutations and structure. A: distribution of missense mutations mapped to the RET protein. Data were based on the curated dataset of cBioportal that contains results from 176 non-redundant studies that have 46,612 samples from 44,284 patients. Locations of the CLD and CRD domains are based on the 3D structure determined by cryo-EM (PDB: 6Q2N) ${ }^{[3]}$; B: structure of RET/ GFR 1/GDNF complex based on PDB 6Q2N. Disulfide bonds in CRD and in GDNF are shown in blue; C: the RET kinase domain structure (PDB: 6NJA) ${ }^{[27]}$. AS: activating segment; V804 and K758: gate residues; Y806: hinge residue; G810: solvent front residue; M918 and A883: two of MTC-associated mutation residues; RET: rearranged during transfection; CLD: cadherin-like domains; CRD: cysteine-rich domain; GDNF: glial cell-derived neurotrophic factor

Cys residues in the CRD domain may actually require the absence of the ligand. It is possible that C630 and C634 located in the linker region between the CRD and the transmembrane domains are in a closer proximity between two RET protein molecules in the receptor/co-receptor/ligand complexes, allowing formation of an intermolecular disulfide bond. However, the extracellular domain of RET or RETC634R could not form dimers in solution ${ }^{[25]}$. It was reported that a fraction of $\mathrm{RET}^{\mathrm{C} 634 \mathrm{R} / \mathrm{Y}}$ expressed in NIH3T3 cells formed dimers under non-reducing conditions ${ }^{[26]}$. Whether the C634R/Y-mediated dimerization is facilitated by a co-receptor, the transmembrane domain, or the intracellular domain remains unknown. Hereditary and somatic RET mutations in the more aggressive MEN2B, such as M918T, A883F, V804M/ Y806C, V804M/E805K, V908M/S904C, are located in the cytoplasmic kinase domain. M918T and S904 are located in the activating segment [Figure $1 \mathrm{C}$ ]. M918T is the strongest transforming mutant in vitro and is associated with the most aggressive tumors ${ }^{[1]}$. V804 is the gatekeeper residue [Figure 1C]. Y806 is a hinge residue in the ATP binding pocket. Mutations in these kinase domain residues may increase ATP binding affinity and/or alter autophosphorylation ${ }^{[5]}$. Mutations in both extracellular and intracellular RET domains are found in FMTC. FMTC-associated mutations may have milder effects on the RET kinase ${ }^{[5,23]}$.

Figure 1A shows the distribution of 701 missense RET mutations from the curated set of 176 nonredundant studies in cBioportal $^{[12,28]}$. These mutations occurred in 413 positions located throughout the molecule. Although these mutations were found in various types of cancers, only a few of these mutations have been annotated functionally. One may speculate that most of these variants of unknown significance 
Table 1. RET fusions in the TCGA curated set of 176 non-redundant studies

\begin{tabular}{|c|c|c|c|c|c|c|c|c|c|c|c|}
\hline \multirow{2}{*}{ RET fusion } & \multicolumn{10}{|c|}{ Tumor type ${ }^{\star}$} & \multirow{2}{*}{ Total } \\
\hline & PTC & LuAd & Thy & Colon & Breast & LuSc & Pro & Ova & Stom & AML & \\
\hline CCDC6-RET & 24 & 4 & 1 & 1 & & & & 1 & 1 & & 32 \\
\hline KIF5B-RET & & 17 & & & & 1 & & & & & 18 \\
\hline NCOA4-RET & 5 & 1 & & 1 & & & 1 & & & & 8 \\
\hline ERC1-RET & 2 & & & & 1 & & & & & & 3 \\
\hline FKBP15-RET & 1 & & & & & & & & & 1 & 2 \\
\hline AKAP13-RET & 1 & & & & & & & & & & 1 \\
\hline DLG5-RET & 1 & & & & & & & & & & 1 \\
\hline PDCD10-RET & & & 1 & & & & & & & & 1 \\
\hline RASSF4-RET & & 1 & & & & & & & & & 1 \\
\hline CUBN-RET & & 1 & & & & & & & & & 1 \\
\hline MRLN-RET & 1 & & & & & & & & & & 1 \\
\hline TBL1XR1-RET & 1 & & & & & & & & & & 1 \\
\hline TFG-RET & & & 1 & & & & & & & & 1 \\
\hline TRIM27-RET & 1 & & & & & & & & & & 1 \\
\hline TRIM33-RET & & 1 & & & & & & & & & 1 \\
\hline SPECC1L-RET & 1 & & & & & & & & & & 1 \\
\hline Subtotal & 38 & 25 & 3 & 2 & 1 & 1 & 1 & 1 & 1 & 1 & 74 \\
\hline
\end{tabular}

*LuAd: lung adenocarcinoma; Thy: poorly differentiated thyroid; LuSc: lung squamous; Pro: prostate; Ova: ovarian; Stom: stomach; AML: acute myeloid leukemia; RET: rearranged during transfection; TCGA: The Cancer Genome Atlas

(VUS) were passenger mutations that did not have a functional role in the tumors. For instance, if a mutated gene was not expressed, the mutation could be considered a silent variant without a function. However, examination of the available expression data of these RET mutants in the tumors in which they were identified showed that most of the mutated RET genes were expressed.

Gene rearrangements that led to fusion RET proteins, on the other hand, were found in $20 \%-40 \%$ of PTC and $1 \%-2 \%$ of lung adenocarcinoma ${ }^{[1,4]}$. Unlike missense substituting mutations that could occur either hereditarily or somatically, and noting that the functional consequences of many mutations are unknown, RET gene fusion occur only somatically and the fusion partners contain protein dimerization domains ${ }^{[4]}$. Consequently, the fusions RET proteins are aberrantly activated via dimerization induced by the fusion partners. Moreover, the fusion partners direct the expression of the fusion oncogenes in those cell types in which RET and/or its co-receptors are not normally expressed ${ }^{[4]}$. This allows oncogenic activation of RET fusions in cell types that the full-length RET mutants cannot accomplish. The fusion partners also alter the cellular location of the RET kinase, allowing fusion kinases to form different signaling hubs ${ }^{[29,30]}$. With exceptions, RET fusions often occur via breakpoints within intron 11, resulting in fusion proteins containing RET intracellular fragments encoded by exons 12-20 (E713 to the C-terminal residue) [Figure 1]. Table 1 lists 74 RET fusions found in 10 tissue types in the curated set of non-redundant studies of cBioportal. Consistent with other reports ${ }^{[4,9,11,31-33]}$, the intrachromosomal rearrangements ${ }^{[34]}$ CCDC6-RET and NCOA4-RET occurred most often in PTC, whereas the intrachromosomal rearrangements KIF5BRET and CCDC6-RET were the most common in lung adenocarcinoma.

Using hybrid capture targeted genomic profiling of RET introns 9-11 and all exons, a study in 9693 cases of breast cancer identified 121 RET alterations ${ }^{[35]}$. Among these were eight activating RET fusions (CCDC6RET, NCOA4-RET, RASGEF1A-RET) and another eight cases of RET gene rearrangements (tandem duplication, intergenic space, and two other cases of potential partner genes). A metastatic, estrogen receptor- and HER2-targeted therapy refractory breast cancer was found to harbor the NCOA4-RET fusion. Combination of the multi-targeted RET TKI cabozantinib in the subsequent treatment regimen resulted in tumor reduction ${ }^{[35,36]}$. 
In addition to analyzing tumor tissue samples, Rich et al. ${ }^{[14]}$ analyzed NGS data of circulating cell-free tumor DNA (cfDNA) from 32,989 patients with metastatic solid tumors (Guardant Health). One hundred seventy-six known and predicted oncogenic alterations were found in 170 patients. These include 143 inframe fusions and 33 missense mutations. In addition, VUS were identified in 529 patients. RET fusions were found in 116 cases of NSCLC, 14 cases of colorectal cancer, 2 cases of breast cancer, 1 case of thyroid cancer, and 3 cases of unknown primary cancer. Among other mutations, M918T and CRD mutations were found in six cases of thyroid cancer and five cases of breast cancer.

\section{RET PROTEIN TYROSINE KINASE INHIBITORS}

\section{Targeting RET fusion ${ }^{+}$NSCLC with multi-targeted TKIs with RET inhibitor activity}

Soon after the discovery of RET fusions in NSCLC ${ }^{[8-11]}$, Drilon et al. ${ }^{[15,37]}$ conducted the first prospective phase 2 clinical trial of treating RET fusion ${ }^{+}$NSCLC patients with cabozantinib. The overall response rate (ORR) to cabozantinib in RET fusion ${ }^{+}$NSCLC was $28 \%$ (7 of 25 patients). This ORR was higher than the ORRs of cabozantinib in unselected NSCLC patients (10\%), the chemotherapy drugs pemetrexed (9\%) and docetaxel (8\%), or the immune checkpoint blockage agent nivolumab (20\%). No co-mutation of known cabozantinib targets was found in these cabozantinib-responsive tumors. However, the ORR was lower than that of the ALK inhibitors (57\%) or ROS1 inhibitors (72\%) in NSCLC patients with ALK or ROS1 fusions. No patient had a complete response to cabozantinib ${ }^{[15]}$. However, in a retrospective analysis of a global, multicenter network registry of 19 RET fusion ${ }^{+}$NSCLC patients, one complete response to cabozantinib (5\%) was recorded ${ }^{[38]}$. Most of the patients (73\%) required dose reduction due to drug-related toxicities. The median progression-free survival (PFS) was 5.5 months. However, durable response was observed in one patient who had been treated with cabozantinib for more than 3 years at the time of reporting.

Two prospective phase 2 trials of vandetanib in RET fusion ${ }^{+}$NSCLC were conducted in East Asia ${ }^{[16,17]}$. The ORR was $18 \%$ in the Korean trial ${ }^{[16]}$, whereas it was $53 \%$ in the Japanese trial ${ }^{[17]}$. The median PFS was 4.5-4.7 months in these two studies. In the global, multicenter registry analysis, the ORR to vandetanib was 18\%, and the median PFS was 2.9 months. Four patients (23\%) required dose reduction in the Korean trial, while 10 patients (53\%) patients required dose reduction in the Japanese trial.

The results of a multicenter, phase 2 trial of lenvatinib in RET fusion ${ }^{+}$NSCLC were reported recently ${ }^{[39]}$. The ORR was $16 \%$ (4/25), and the median PFS was 7.3 months. Twenty-three (92\%) patients had a drugrelated adverse event and six (24\%) patients discontinued the lenvatinib treatment due to drug-related toxicities. Responses to other multi-targeted TKIs sunitinib, alectinib, and nintedanib in RET fusion ${ }^{+}$ NSCLC were documented in analyses of retrospective clinical data ${ }^{[38,40]}$. Interestingly, in addition to the above mentioned complete response of a patient to cabozantinib treatment, one patient who received nintedanib had complete response ${ }^{[38]}$. Furthermore, stable disease was observed in patients treated with sorafenib and ponatinib ${ }^{[38,40]}$.

It is believed that the adverse events induced by the multi-targeted RET TKIs were in part due to VEGFR inhibition ${ }^{[4,15]}$. For instance, cabozantinib inhibits RET with an $\mathrm{IC}_{50}$ of $5.2 \mathrm{nM}$, but it inhibits VEGFR2 with an $\mathrm{IC}_{50}$ of $0.04 \mathrm{nM}$. Agerafenib (RXDX-105) was identified as a multi-targeted RET TKI ( $\mathrm{IC}_{50}: 0.33$ $\mathrm{nM})$ that spared VEGFR1 $\left(\mathrm{IC}_{50}: 140 \mathrm{nM}\right)$ and VEGFR2 $\left(\mathrm{IC}_{50}: 257 \mathrm{nM}\right)^{[41,42]}$. Agerafenib was originally identified as a BRAF inhibitor but kinase profiling revealed its RET inhibitor activity. In a phase I/Ib clinical trial of agerafenib, the recommended phase 2 dose was selected at $275 \mathrm{mg}$ once a day. At this dose, agerafenib had a steady state plasma level above the target thresholds of inhibiting RET and BRAF, but below that of inhibiting VGEFR $2^{[43]}$. In the phase Ib cohort, no response was observed in prior RET inhibitor (cabozantinib, vandetanib)-treated patients. In RET inhibitor-naïve NSCLC patients, the ORR was $19 \%(6 / 31)$ and stable disease was achieved in 39\% (12/31) of patients. Notably, none of the responders 
had KIF5B-RET fusion although stable disease was achieved in agerafenib-treated patients with KIF5BRET fusion ${ }^{+}$tumors. In non-KIF5B RET fusion tumors, the ORR was 67\%. The mechanism underlying the difference in response to agerafenib between KIF5B-RET fusion and non-KIF5B RET fusion remains elusive.

\section{RET-selective kinase inhibitors}

Pralsetinib and selpercatinib are the new generation of RET-selective TKIs that were recently developed and rapidly progressed to clinical trials ${ }^{[18,19]}$. Pralsetinib has 87 -fold selectivity against VEGFR2 and 20 -fold selectivity against JAK $1{ }^{[18,20]}$. Selpercatinib has $>100$-fold selectivity against VEGFR $2^{[44]}$. In addition to being highly selective for RET, both pralsetinib and selpercatinib are effective in inhibiting the $\mathrm{RET}^{\mathrm{V} 804 \mathrm{~L} / \mathrm{M}}$ gatekeeper mutants ${ }^{[18-20,44]}$. Another important property of these drugs is that they are effective in the central nervous system. Intracranial metastases occur frequently in RET fusion ${ }^{+}$NSCLC $^{[40]}$.

In the phase $1 / 2$ trial of pralsetinib (NCT03037385) in patients with thyroid, NSCLC, and other advanced solid tumors (ARROW), treatment-related adverse events were mostly grade $1 / 2$ at the chosen phase 2 dose of $400 \mathrm{mg}$ QD. The ORR was $58 \%$ and the disease control rate was $96 \%$ in RET fusion ${ }^{+}$NSCLC patients $(n=48)$ regardless of prior treatments (chemotherapy, PD-1 or PD-L1 inhibitor, multi-targeted TKIs with RET inhibitor activity $)^{[20]}$. In MTC $(n=32)$, the ORR was $56 \%$ and the disease control rate was $94 \%{ }^{[45]}$. In PTC, pralsetinib had a 83\% ORR $(n=6)^{[45]}$. Responses were also seen in RET fusion ${ }^{+}$pancreatic cancer and intrahepatic bile duct carcinoma ${ }^{[20]}$. In the phase $1 / 2$ trial of selpercatinib (NCT03157128) in patients with RET fusion ${ }^{+}$solid tumors (LIBRETTO-001), a recommended phase 2 dose of $160 \mathrm{mg}$ twice a day was established. Treatment-related toxicity was mostly in the low grade. Preliminary data from the selpercatinib trial showed an ORR of $68 \%$ in RET fusion ${ }^{+}$NSCLC $(n=105)$. In treatment naïve RET fusion $^{+}$NSCLC, the ORR was $85 \%$. The median duration of response was 20.3 months. The responses to pralsetinib and selpercatinib were observed in tumors with all RET fusion partners ${ }^{[20,21,45]}$. Thus, the results from pralsetinib and selpercatinib trials showed that these RET-specific TKIs gave high response rates comparable to those of ALK- and ROS1 fusion-targeted therapies in NSCLC at the tolerated doses and the responses were more durable than that of the multi-targeted TKIs with RET inhibitor activity.

\section{RET MUTATIONS RESISTANT TO MULTI-TARGETED AND SPECIFIC RET TYROSINE KINASE INHIBITORS}

\section{RET mutations resistant to multi-targeted RET TKIs}

A lesson learned from PTK-targeted therapies in chronic myeloid leukemia (BCR-ABL) ${ }^{[46-48]}$ and NSCLC (EGFR mutants, ALK and ROS1 fusions) ${ }^{[49-53]}$ is that the secondary mutations in the targeted PTK can cause resistance to the TKIs. By aligning MEN2/MTC-associated RET mutations with imatinib-resistant BCR-ABL residues, Carlomagno et al.$^{[54,55]}$ found that the $\mathrm{RET}^{\mathrm{V} 804 \mathrm{~L} / \mathrm{M}}$ mutants at the gatekeeper residue and the $\mathrm{RET}^{\mathrm{Y} 806 \mathrm{C}}$ mutant at the hinge residue were resistant to vandetanib. Using the BaF3/KIF5B-RET cell model, we identified 14 RET kinase domain mutations resistant to cabozantinib, vandetanib, lenvatinib, and/or nintedanib ${ }^{[27,56,57]}$. These mutations were located in both drug-binding pockets and in the C-lobe outside of the drug-binding pocket. V804L/M mutations at the gatekeeper residue and Y806N mutation at the hinge region were pan-resistant to these drugs. Mutations at the C-lobe solvent-front G810 and N-lobe solvent front L730 residues had different profiles of resistance to these drugs depending on the substituting amino acid residues. Of particular interest was that the solvent-front RET ${ }^{\mathrm{G} 810 \mathrm{~A}}$ variant, which was isolated as a vandetanib-resistant mutation, have gained slight sensitivity to the known type II inhibitor ponatinib and cabozantinib ${ }^{[56,57]}$. Two acquired vandetanib-resistant RET kinase domain mutation cases have been reported in NSCLC. The first was a CCDC6-RET fusion ${ }^{+}$metastatic lung adenocarcinoma that developed adaptive resistance to vandetanib ${ }^{[58]}$. The resistant tumor had an acquired CCDC6-RET ${ }^{\text {S904F }}$ mutation. It was determined that the activating loop S904F mutation caused an allosteric effect that enhanced the basal 
kinase activity. The second reported case was a patient with CCDC6-RET fusion ${ }^{+}$metastatic NSCLC ${ }^{[59]}$. The liver metastases responded to vandetanib initially, but progressed after 10 months. cfDNA analysis detected the presence of the CCDC6-RET ${ }^{\mathrm{V} 804 \mathrm{M}}$ gatekeeper mutation. In addition, a MTC patient with $\mathrm{RET}^{\mathrm{M} 918 \mathrm{~T}}$ tumor was treated sequentially with sorafenib, vandetanib, cabozantinib, MGCD-516 and agerafenib. After disease progressed, a cfDNA assay revealed acquired $\mathrm{RET}^{\mathrm{V} 804 \mathrm{M}}$ gatekeeper mutation ${ }^{[19]}$.

\section{RET mutations resistant to RET-specific TKIs}

As mentioned above, the potent, RET-specific TKIs pralsetinib and selpercatinib are effective against the $\mathrm{RET}^{\mathrm{V} 804 \mathrm{~L} / \mathrm{M}}$ gatekeeper mutants. An in vitro RET kinase assay showed that selpercatinib was ineffective against the $\mathrm{RET}^{\mathrm{G} 810 \mathrm{R}}$ solvent front mutant ${ }^{[60]}$. $\mathrm{RET}^{\mathrm{G} 810}$ residue is at the paralogous position of the C-lobe solvent front residue in $\mathrm{EGFR}^{\mathrm{G} 796}, \mathrm{ALK}^{\mathrm{G} 1202}, \mathrm{ROS}_{1}^{\mathrm{G} 2032}$ and $\mathrm{NTRK}_{1}^{\mathrm{G} 595} / \mathrm{NTRK}^{\mathrm{G} 623}$. The $\mathrm{EGFR}^{\mathrm{G} 796 / \mathrm{R}}$ mutants are resistant to the third-generation, EGFR ${ }^{\mathrm{T} 790 \mathrm{M}}$-sensitive EGFR inhibitor osimertinib ${ }^{[6,62]}$. The ALK ${ }^{\mathrm{G} 1202 \mathrm{R}}$ mutation was found in $21 \%-43 \%$ of acquired ALK mutations resistant to the second-generation, ALK ${ }^{\mathrm{L} 1196 \mathrm{M}}$ gatekeeper mutant-sensitive ALK TKIs certinib, alectinib and brigatinib ${ }^{[63,64]}$. ROS1 ${ }^{\mathrm{G} 2032 \mathrm{R}}$ and the adjacent $\mathrm{ROS}_{1}{ }^{\mathrm{D} 2033 \mathrm{~N}}$ mutations are resistant to crizotinib ${ }^{[65]}$. NTRK1 ${ }^{\mathrm{G} 595 \mathrm{R}} / \mathrm{NTRK} 3{ }^{\mathrm{G} 623 \mathrm{R}}$ are resistant to entrectinib and larotrectinib ${ }^{[66]}$. Recently, we identified $\mathrm{RET}^{\mathrm{G} 810 \mathrm{C} / / \mathrm{R}}$ as selpercatinib-resistant mutations in a preclinical study ${ }^{[67]}$. Consistent with our preclinical finding, $\mathrm{RET}^{\mathrm{G} 810 \mathrm{C} / \mathrm{S} / \mathrm{R}}$ mutations were reported in a case of a KIF5B-RET fusion NSCLC and a RET ${ }^{\mathrm{G} 810 \mathrm{C}}$ mutation was reported in a case CCDC6-RET fusion NSCLC; both had adaptive resistant to selpercatinib ${ }^{[22]}$.

\section{CONCLUSIONS}

Except for MTC and PTC, which have high rates of RET alterations, RET mutations and gene rearrangements have been observed at low frequencies in many types of human cancers. Gene rearrangements result in RET fusion oncogenes. Aside from a few well-characterized MTC-associated RET mutations, such as the $\mathrm{RET}^{\mathrm{C} 634 \mathrm{R} / \mathrm{Y}}$ and $\mathrm{RET}^{\mathrm{M} 918 \mathrm{~T}}$ variants, most of the recently identified RET mutations are of VUS. Moreover, the activating mechanisms of many MTC-associated RET mutations remain incompletely elucidated. For instance, it is believed that mutations of cysteine residues in the CRD lead to receptor dimerization via formation of intermolecular disulfide bonds ${ }^{[4]}$. However, experimental evidence to support this notion has yet to be reported.

The high ORR of RET-selective TKIs pralsetinib and selpercatinib in RET-altered cancers unequivocally demonstrates the RET dependence of tumors carrying these RET oncogenes. This observation has led to the proposal of molecular classification of RET-driven tumors as "retoma" ${ }^{\text {"[68] }}$. In our view, RET fusion ${ }^{+}$ tumors could all be classified as retoma. Tumors with the RET ${ }^{\mathrm{M} 918 \mathrm{~T}}$ mutant should be included as retoma because these tumors responded to pralsetinib and selpercatinib in clinical studies. Inclusion of tumors with other RET mutations in the absence of RET chromosomal rearrangement as retoma will await preclinical and clinical evaluations of these mutations.

It is anticipated that the potent RET-selective TKIs pralsetinib and selpercatinib will be approved by the United States FDA soon and thus become the first two approved RET-targeted therapy drugs. However, adaptive RET mutants resistant to selpercatinib have been found. Similar or novel RET mutants resistant to pralsetinib are predicted in addition to resistance caused by oncogene bypass mechanisms. Oncogene bypass mechanisms in solid tumors are multiple and may be influenced by tissue-specific tumor microenvironment. Intensive investigation is needed to overcome these targeted protein tyrosine kinase-bypass resistance mechanisms. For fusion protein tyrosine kinase-driven NSCLC that respond to potent, target-selective TKIs, on-target mutations appear to be a major adaptive resistance mechanism. While pralsetinib and selpercatinib are effective against the RET gatekeeper mutants, a lesson learned from protein tyrosine kinase-targeted therapies in NSCLC is that non-gatekeeper mutations will cause resistance to the new generations of TKIs. Pralsetinib and selpercatinib are no exception. Fortunately, 
TKIs of different chemical scaffolds can be developed to inhibit adaptive kinase mutants ${ }^{[63,69]}$. Therefore, the research and the pharmaceutical communities should continue to develop pipelines of RET-specific inhibitors. Pralsetinib and selpercatinib are an important milestone, but not the end of the journey, in developing RET-targeted therapy for retoma.

\section{DECLARATIONS}

\section{Acknowledgments}

We thank Kathy Kyler for editing the manuscript.

\section{Authors' contributions}

Review the literature: Liu X, Hu X, Wu J

Analyzed genomics and structural biology data: $\mathrm{Hu}$ X, Mooers BHM, Wu J

Drafted the article: Wu J

Writing or editing the article: Liu X, Hu X, Shen T, Li Q, Mooers BHM, Wu J

\section{Availability of data and materials}

Not applicable.

\section{Financial support and sponsorship}

This work was supported by a Presbyterian Health Foundation Grant (to Mooers BHM, Wu J) and by National Natural Science Foundation of China (No. 81904130 to Hu X, Li Q).

\section{Conflicts of interest}

All authors declared that there are no conflicts of interest.

\section{Ethical approval and consent to participate}

Not applicable.

\section{Consent for publication}

Not applicable.

\section{Copyright}

(c) The Author(s) 2020.

\section{REFERENCES}

1. Mulligan LM. RET revisited: expanding the oncogenic portfolio. Nat Rev Cancer 2014;14:173-86.

2. Roskoski R Jr, Sadeghi-Nejad A. Role of RET protein-tyrosine kinase inhibitors in the treatment RET-driven thyroid and lung cancers. Pharmacol Res 2018;128:1-17.

3. Li J, Shang G, Chen YJ, Brautigam CA, Liou J, et al. Cryo-EM analyses reveal the common mechanism and diversification in the activation of RET by different ligands. Elife 2019;8.

4. Drilon A, Hu ZI, Lai GGY, Tan DSW. Targeting RET-driven cancers: lessons from evolving preclinical and clinical landscapes. Nat Rev Clin Oncol 2018;15:151-67.

5. Krampitz GW, Norton JA. RET gene mutations (genotype and phenotype) of multiple endocrine neoplasia type 2 and familial medullary thyroid carcinoma. Cancer 2014;120:1920-31.

6. Santoro M, Carlomagno F, Romano A, Bottaro DP, Dathan NA, et al. Activation of RET as a dominant transforming gene by germline mutations of MEN2A and MEN2B. Science 1995;267:381-3.

7. Grieco M, Santoro M, Berlingieri MT, Melillo RM, Donghi R, et al. PTC is a novel rearranged form of the ret proto-oncogene and is frequently detected in vivo in human thyroid papillary carcinomas. Cell 1990;60:557-63.

8. Ju YS, Lee WC, Shin JY, Lee S, Bleazard T, et al. A transforming KIF5B and RET gene fusion in lung adenocarcinoma revealed from whole-genome and transcriptome sequencing. Genome Res 2012;22:436-45.

9. Kohno T, Ichikawa H, Totoki Y, Yasuda K, Hiramoto M, et al. KIF5B-RET fusions in lung adenocarcinoma. Nat Med 2012;18:375-7.

10. Lipson D, Capelletti M, Yelensky R, Otto G, Parker A, et al. Identification of new ALK and RET gene fusions from colorectal and 
lung cancer biopsies. Nat Med 2012;18:382-4.

11. Takeuchi K, Soda M, Togashi Y, Suzuki R, Sakata S, et al. RET, ROS1 and ALK fusions in lung cancer. Nat Med 2012;18:378-81.

12. Gao J, Aksoy BA, Dogrusoz U, Dresdner G, Gross B, et al. Integrative analysis of complex cancer genomics and clinical profiles using the cBioPortal. Sci Signal 2013;6:pl1.

13. Kato S, Subbiah V, Marchlik E, Elkin SK, Carter JL, et al. RET Aberrations in Diverse Cancers: Next-Generation Sequencing of 4,871 Patients. Clin Cancer Res 2017;23:1988-97.

14. Rich TA, Reckamp KL, Chae YK, Doebele RC, Iams WT, et al. Analysis of cell-free DNA from 32,989 advanced cancers reveals novel co-occurring activating RET alterations and oncogenic signaling pathway aberrations. Clin Cancer Res 2019;25:5832-42.

15. Drilon A, Rekhtman N, Arcila M, Wang L, Ni A, et al. Cabozantinib in patients with advanced RET-rearranged non-small-cell lung cancer: an open-label, single-centre, phase 2, single-arm trial. Lancet Oncol 2016;17:1653-60.

16. Lee SH, Lee JK, Ahn MJ, Kim DW, Sun JM, et al. Vandetanib in pretreated patients with advanced non-small cell lung cancerharboring RET rearrangement: a phase II clinical trial. Ann Oncol 2017;28:292-7.

17. Yoh K, Seto T, Satouchi M, Nishio M, Yamamoto N, et al. Vandetanib in patients with previously treated RET-rearranged advanced non-small-cell lung cancer (LURET): an open-label, multicentre phase 2 trial. Lancet Respir Med 2017;5:42-50.

18. Subbiah V, Gainor JF, Rahal R, Brubaker JD, Kim JL, et al. Precision Targeted Therapy with BLU-667 for RET-Driven Cancers. Cancer Discov 2018;8:836-49.

19. Subbiah V, Velcheti V, Tuch BB, Ebata K, Busaidy NL, et al. Selective RET kinase inhibition for patients with RET-altered cancers. Ann Oncol 2018;29:1869-76.

20. Gainor JF, Lee DH, Curijgliano G, Doebele RC, Kim CSB, et al. Clinical activity and tolerability of BLU-667, a highly potent and selective RET inhibitor, in patients (pts) with advanced RET-fusion+ non-small cell lung cancer (NSCLC). ASCO Annual Meeting 2019: Abstract 9008.

21. Drilon A, Oxnard GR, Wirth L, Besse B, Gautschi O, et al. PL02.08: registrational results of LIBRETTO-001: a Phase 1/2 Trial of LOXO-292 in patients with RET fusion-positive lung cancers. J Thorac Oncol 2019;14:S6-S7.

22. Solomon BJ, Tan L, Lin JJ, Wong SQ, Hollizeck S, et al. RET solvent front mutations mediate acquired resistance to selective RET inhibition in RET-driven malignancies. J Thorac Oncol 2020;15:541-9.

23. Wells SA Jr, Pacini F, Robinson BG, Santoro M. Multiple endocrine neoplasia type 2 and familial medullary thyroid carcinoma: an update. J Clin Endocrinol Metab 2013;98:3149-64.

24. Plaza-Menacho I, Barnouin K, Goodman K, Martinez-Torres RJ, Borg A, et al. Oncogenic RET kinase domain mutations perturb the autophosphorylation trajectory by enhancing substrate presentation in trans. Mol Cell 2014;53:738-51.

25. Goodman KM, Kjaer S, Beuron F, Knowles PP, Nawrotek A, et al. RET recognition of GDNF-GFRalphal ligand by a composite binding site promotes membrane-proximal self-association. Cell Rep 2014;8:1894-904.

26. Asai N, Iwashita T, Matsuyama M, Takahashi M. Mechanism of activation of the ret proto-oncogene by multiple endocrine neoplasia 2A mutations. Mol Cell Biol 1995;15:1613-9.

27. Terzyan SS, Shen T, Liu X, Huang Q, Teng P, et al. Structural basis of resistance of mutant RET protein-tyrosine kinase to its inhibitors nintedanib and vandetanib. J Biol Chem 2019;294:10428-37.

28. Cerami E, Gao J, Dogrusoz U, Gross BE, Sumer SO, et al. The cBio cancer genomics portal: an open platform for exploring multidimensional cancer genomics data. Cancer Discov 2012;2:401-4.

29. Levinson S, Cagan RL. Drosophila cancer models identify functional differences between ret fusions. Cell Rep 2016;16:3052-61.

30. Das TK, Cagan RL. KIF5B-RET oncoprotein signals through a multi-kinase signaling hub. Cell Rep 2017;20:2368-83.

31. Gainor JF, Shaw AT. Novel targets in non-small cell lung cancer: ROS1 and RET fusions. Oncologist 2013;18:865-75.

32. Tsuta K, Kohno T, Yoshida A, Shimada Y, Asamura H, et al. RET-rearranged non-small-cell lung carcinoma: a clinicopathological and molecular analysis. Br J Cancer 2014;110:1571-8.

33. Wang R, Hu H, Pan Y, Li Y, Ye T, et al. RET fusions define a unique molecular and clinicopathologic subtype of non-small-cell lung cancer. J Clin Oncol 2012;30:4352-9.

34. Mizukami T, Shiraishi K, Shimada Y, Ogiwara H, Tsuta K, et al. Molecular mechanisms underlying oncogenic RET fusion in lung adenocarcinoma. J Thorac Oncol 2014;9:622-30.

35. Paratala BS, Chung JH, Williams CB, Yilmazel B, Petrosky W, et al. RET rearrangements are actionable alterations in breast cancer. Nat Commun 2018;9:4821

36. Nigro CL, Rusmini M, Ceccherini I. RET in breast cancer: pathogenic implications and mechanisms of drug resistance. Cancer Drug Resist 2019;2:1136-52.

37. Drilon A, Wang L, Hasanovic A, Suehara Y, Lipson D, et al. Response to Cabozantinib in patients with RET fusion-positive lung adenocarcinomas. Cancer Discov 2013;3:630-5.

38. Gautschi O, Milia J, Filleron T, Wolf J, Carbone DP, et al. Targeting RET in patients with RET-rearranged lung cancers: results from the global, multicenter RET registry. J Clin Oncol 2017;35:1403-10.

39. Hida T, Velcheti V, Reckamp KL, Nokihara H, Sachdev P, et al. A phase 2 study of lenvatinib in patients with RET fusion-positive lung adenocarcinoma. Lung Cancer 2019;138:124-30.

40. Drilon A, Lin JJ, Filleron T, Ni A, Milia J, et al. Frequency of brain metastases and multikinase inhibitor outcomes in patients with RET-rearranged lung cancers. J Thorac Oncol 2018;13:1595-601.

41. James J, Ruggeri B, Armstrong RC, Rowbottom MW, Jones-Bolin S, et al. CEP-32496: a novel orally active BRAF(V600E) inhibitor with selective cellular and in vivo antitumor activity. Mol Cancer Ther 2012;11:930-41.

42. Li GG, Somwar R, Joseph J, Smith RS, Hayashi T, et al. Antitumor activity of RXDX-105 in multiple cancer types with RET rearrangements or mutations. Clin Cancer Res 2017;23:2981-90.

43. Drilon A, Fu S, Patel MR, Fakih M, Wang D, et al. A phase I/Ib trial of the VEGFR-sparing multikinase RET inhibitor RXDX-105. 
Cancer Discov 2019;9:384-95.

44. Brandhuber BJ, Haas J, Tuch B, Ebata K, Bouhana K, et al. The development of a potent, KDR/VEGFR2-sparing RET kinase inhibitor for treating patients with RET-dependent cancers. Eur J Cancer 2016;69:S144.

45. Taylor MH, Gainor JF, Hu MI, Zhu VW, Lopes G, et al. Activity and tolerability of BLU-667, a highly potent and selective RET inhibitor, in patients with advanced RET-altered thyroid cancers. J Clin Oncol 2019;37:Abstract 6018.

46. Shah NP, Nicoll JM, Nagar B, Gorre ME, Paquette RL, et al. Multiple BCR-ABL kinase domain mutations confer polyclonal resistance to the tyrosine kinase inhibitor imatinib (STI571) in chronic phase and blast crisis chronic myeloid leukemia. Cancer Cell $2002 ; 2: 117-25$.

47. Hochhaus A, Kreil S, Corbin AS, La Rosee P, Muller MC, et al. Molecular and chromosomal mechanisms of resistance to imatinib (STI571) therapy. Leukemia 2002;16:2190-6.

48. Gorre ME, Mohammed M, Ellwood K, Hsu N, Paquette R, et al. Clinical resistance to STI-571 cancer therapy caused by BCR-ABL gene mutation or amplification. Science 2001;293:876-80.

49. Riely GJ, Yu HA. EGFR: The paradigm of an oncogene-driven lung cancer. Clin Cancer Res 2015;21:2221-6.

50. Katayama R, Shaw AT, Khan TM, Mino-Kenudson M, Solomon BJ, et al. Mechanisms of acquired crizotinib resistance in ALKrearranged lung Cancers. Sci Transl Med 2012;4:120ra17.

51. Katayama R, Lovly CM, Shaw AT. Therapeutic targeting of anaplastic lymphoma kinase in lung cancer: a paradigm for precision cancer medicine. Clin Cancer Res 2015;21:2227-35.

52. Gainor JF, Shaw AT. Emerging paradigms in the development of resistance to tyrosine kinase inhibitors in lung cancer. J Clin Oncol 2013;31:3987-96.

53. Awad MM, Katayama R, McTigue M, Liu W, Deng YL, et al. Acquired resistance to crizotinib from a mutation in CD74-ROS1. N Engl J Med 2013;368:2395-401.

54. Carlomagno F, Guida T, Anaganti S, Vecchio G, Fusco A, et al. Disease associated mutations at valine 804 in the RET receptor tyrosine kinase confer resistance to selective kinase inhibitors. Oncogene 2004;23:6056-63.

55. Carlomagno F, Guida T, Anaganti S, Provitera L, Kjaer S, et al. Identification of tyrosine 806 as a molecular determinant of RET kinase sensitivity to ZD6474. Endocr Relat Cancer 2009;16:233-41.

56. Huang Q, Schneeberger VE, Luetteke N, Jin C, Afzal R, et al. Preclinical Modeling of KIF5B-RET Fusion Lung Adenocarcinoma. Mol Cancer Ther 2016;15:2521-9.

57. Liu X, Shen T, Mooers BHM, Hilberg F, Wu J. Drug resistance profiles of mutations in the RET kinase domain. Br J Pharmacol 2018;175:3504-15.

58. Nakaoku T, Kohno T, Araki M, Niho S, Chauhan R, et al. A secondary RET mutation in the activation loop conferring resistance to vandetanib. Nat Commun 2018;9:625.

59. Dagogo-Jack I, Stevens SE, Lin JJ, Nagy R, Ferris L, et al. Emergence of a RET V804M Gatekeeper mutation during treatment with Vandetanib in RET-rearranged NSCLC. J Thorac Oncol 2018;13:e226-e7.

60. Andrews SW, Aronow S, Blake JF, Brandhuber BJ, Cook A, et al. Substituted pyrazol[1,5-A]pyridine compounds as RET kinase inhibitors. WO2018/071447 A1 2017.

61. Ou SI, Cui J, Schrock AB, Goldberg ME, Zhu VW, et al. Emergence of novel and dominant acquired EGFR solvent-front mutations at Gly796 (G796S/R) together with C797S/R and L792F/H mutations in one EGFR (L858R/T790M) NSCLC patient who progressed on osimertinib. Lung Cancer 2017;108:228-31.

62. Yang Z, Yang N, Ou Q, Xiang Y, Jiang T, et al. Investigating novel resistance mechanisms to third-generation EGFR tyrosine kinase inhibitor osimertinib in non-small cell lung cancer patients. Clin Cancer Res 2018;24:3097-107.

63. Gainor JF, Dardaei L, Yoda S, Friboulet L, Leshchiner I, et al. Molecular mechanisms of resistance to first- and second-generation ALK inhibitors in ALK-rearranged lung cancer. Cancer Discov 2016;6:1118-33.

64. Zhang S, Anjum R, Squillace R, Nadworny S, Zhou T, et al. The potent ALK inhibitor Brigatinib (AP26113) overcomes mechanisms of resistance to first- and second-generation ALK inhibitors in preclinical models. Clin Cancer Res 2016;22:5527-38.

65. Gainor JF, Tseng D, Yoda S, Dagogo-Jack I, Friboulet L, et al. Patterns of metastatic spread and mechanisms of resistance to Crizotinib in ROS1-positive non-small-cell lung cancer. JCO Precis Oncol 2017;2017.

66. Fuse MJ, Okada K, Oh-Hara T, Ogura H, Fujita N, et al. Mechanisms of resistance to NTRK inhibitors and therapeutic strategies in NTRK1-rearranged cancers. Mol Cancer Ther 2017;16:2130-43.

67. Shen T, Terzyan SS, Liu X, Mooers BHM, Wu J. Structural insight into sensitivity and resistance of RET mutants to selpercatinib (LOXO-292). Sixth AACR-IASLC International Joint Conference: Lung Cancer Translational Science from the Bench to the Clinic 2020;Abstract:B18.

68. Kohno T, Tabata J, Nakaoku T. REToma: a cancer subtype with a shared driver oncogene. Carcinogenesis 2019; Epub ahead of print. doi: 10.1093/carcin/bgz184.

69. Drilon A, Ou SI, Cho BC, Kim DW, Lee J, et al. Repotrectinib (TPX-0005) is a next-generation ROS1/TRK/ALK inhibitor that potently inhibits ROS1/TRK/ALK solvent- front mutations. Cancer Discov 2018;8:1227-36. 